www.nature.com/gt

\title{
Editorial
}

\section{Prospects for gene therapy of diabetes mellitus}

Diabetes mellitus is usually classified as type 1 or type 2 diabetes. Type 1 results from a $\beta$-cell defect, often due to an autoimmune process. Type 2 diabetes is characterized by insulin resistance which is often combined with an insulin secretory defect. The number of people suffering from diabetes is growing at an alarming rate. In 1997, the estimated number of people worldwide with diabetes was 124 million, 97\% of these having type 2 diabetes. $^{1}$ Based on estimates of changes in lifestyle, economic development and population figures, the worldwide prevalence of diabetes mellitus by the year 2010 is projected to reach 221 million people. ${ }^{1}$ Since severe complications can occur if the disease is not under strict glycemic control, it is evident that the epidemic of diabetes is becoming an enormous health problem. In light of this disturbing scenario, not only are efforts to promote exercise and low fat intake of paramount importance, but also new techniques that can restore metabolic control in a safe and cost-efficient manner must be developed. One such approach, which might be useful, is gene therapy. Using a broad definition of gene therapy, it is possible to outline quite a few gene transfer strategies that could possibly help diabetics. ${ }^{2}$

The first gene therapy approach to diabetes was put forward shortly after the cloning of the insulin gene. It was proposed that non-insulin producing cells could be made into insulin-producing $\beta$ cells using a suitable promoter and insulin gene construct, and that these substitute cells could restore insulin production in type 1 and some type 2 diabetics. The original experiments were carried out on cell lines and fibroblasts, but since then hepatocytes, myocytes, pituitary and exocrine cells have also been made into artificial insulin-producing cells. Unfortunately, none of these cells respond to glucose with physiological secretion of insulin. Instead, it is only possible to achieve regulation of insulin gene transcription by using promoter constructs that respond to glucose. Because transcription is a much slower process than regulated release from secretory granules, there is a substantial risk of the insulin production getting out of phase with fluctuations in glucose levels leading to episodes of severe hypoglycemia. Thus, the generation of a substitute $\beta$ cell from non- $\beta$ cells may prove to be exceedingly difficult.

Another gene therapy approach aims at genetically manipulating $\beta$ cells so that they produce a local $\beta$ cell protection factor. In individuals in whom autoimmune destruction of $\beta$ cells has begun, but not reached the end stage, it would make sense to rescue the remaining $\beta$ cells by such a gene therapy approach. Assuming that it is possible to target a vector to the $\beta$ cell in vivo, the resulting $\beta$ cell production of a local survival factor would not only save the $\beta$ cells, it would also leave the immune system in general unaffected as the transgene production is localized to the islets. This strategy was first proposed in a study which demonstrated that transgene production of interleukin-1 receptor antagonist protein desensitized the $\beta$ cells to interleukin-1 induced nitric oxide production. ${ }^{3}$ It is possible that $\beta$ cells are destroyed in type 1 diabetes as a result of macrophagemediated release of cytokines and nitric oxide. It might also be cytotoxic $\mathrm{T}$ cells that kill the $\beta$ cell by releasing the apoptotic signals perforin and Fas ligand. In both cases, quite a few $\beta$ cell survival factors have been envisaged. ${ }^{4}$ In addition to cytokine antagonists such as the interleukin-1 receptor antagonist, immune modulators such as TGF- $\beta$ and CGRP, inhibitors of Fas ligand signalling, anti-apoptotic factors such as Bcl-2 and A20, and anti-stress factors such as thioredoxin all qualify as interesting candidates. ${ }^{4,5}$ These factors have been addressed experimentally and could possibly, when expressed by the $\beta$ cells, promote $\beta$ cell survival.

Insulin-producing cells can not only be manipulated for the avoidance of autoimmune destruction, but also for transplantation purposes. Transplantation of human or pig islets to diabetic recipients is problematic due to poor grafting and rejection. To promote successful grafting, islets could possibly be transduced ex vivo to produce heme oxygenase and vascular endothelium growth factor. These proteins protect against hypoxia and stimulate vascular neogenesis. Rejection of allografts and xenografts are highly complex processes. However, one step forward was taken when transgenic pigs were generated, which expressed a human complement regulatory protein (hDAF). This protein attenuates the antibodymediated complement activation, thereby lessening the problem with hyperacute rejection. Attempts to manipulate pigs genetically not to express the alpha Gal epitope are also underway. ${ }^{6}$

However, the greatest problem right now for all $\beta$ cell transduction strategies is the lack of efficient and safe vectors. To transduce $\beta$ cells in vivo, the vector would have to be obtainable in large quantities, stable when administered in vivo, reach the $\beta$ cells from the blood stream and efficiently and selectively transduce the nonreplicating $\beta$ cell. All this would have to be achieved without inducing toxicity, immune reactions or pathological recombinations. Although considerable improvements in vector design have been accomplished, there is 
a long way to go. The lentivirus, which transduces $\beta$ cells in vitro, is derived from the HIV-1 virus, which might preclude its use in humans due to the risk of pathological recombinations. More promising is perhaps the adenovirus, which transduces human islet cells not only in vitro, but also ex vivo. Indeed, intra-arterial injection of adenovirus into a whole human pancreas resulted in transduction of $50 \%$ of the $\beta$ cells. ${ }^{7}$ This finding gives hope for the future, but gene transfer techniques need to be developed that can achieve therapeutic long-term expression, in vivo regulation of transgene expression and lack of immune triggering, in order to conduct gene therapy on pancreatic $\beta$ cells in vivo.

The technical problems associated with the transfection of $\beta$ cells are possibly avoided by using the DNA vaccination approach. In individuals with a high risk of developing diabetes, as indicated by genetic and humoral markers, but who have not yet entered the phase of autoimmune $\beta$ cell destruction, it might be possible to prevent the progression of the disease by DNA vaccination. In mice, it has already been observed that DNA vaccination with a glutamic acid decarboxylase (GAD) gene construct generates a humoral immune response. ${ }^{\circ}$ GAD is considered a key autoantigen in type 1 diabetes, and if the DNA vaccination approach leads to tolerization, $\beta$ cell destruction might be avoided. DNA vaccination might also be used for inducing immunity against key factors that mediate the inflammatory process. For example, DNA vaccination with naked DNA encoding C-C chemokines has been observed to protect against experimental autoimmune encephalomyelitis. ${ }^{9}$

Finally, with increasing knowledge of the factors that control $\beta$ cell differentiation and replication, a genetic approach that stimulates the regeneration of the $\beta$ cell mass becomes feasible. For a long time, the molecular control of $\beta$ cell growth and differentiation has been obscure. However, the Edlund group in Sweden has recently demonstrated that the transcription factor IPF1 participates in maintaining the $\beta$ cell phenotype and euglycemia in vivo. ${ }^{10}$ Moreover, they have also reported that Notch signaling controls the decision between pancreatic exocrine and pancreatic endocrine differentiation. ${ }^{11}$ These important findings could make way for large-scale production of $\beta$ cells intended for transplantation to diabetics, or for in situ regeneration of $\beta$ cells in diabetics. However, such an approach must be combined with a strategy to prevent autoimmune destruction of the newly formed $\beta$ cells.

Although significant progress is being made in this field, it should be emphasized that gene therapy is neither a low cost benefit nor a low risk benefit approach.
For example, there is the obvious risk that the gene therapy could induce severe hypoglycemia if the number of gene-modified insulin-producing cells and their insulin release is not under perfect control. Because type 1 diabetes is usually more severe than type 2 , it is natural to assume that the first gene therapy efforts will be dedicated to the treatment of type 1, and that the experiences from these trials will be used for later strategies for the treatment of type 2. Thus, it must be clear that the benefits of gene therapy of diabetes outweigh the risks and offer advantages as compared with conventional treatment before this approach could become accepted in general practice.

\section{$\mathrm{N}$ Welsh \\ Department of Medical Cell Biology Uppsala University S-751 23 Uppsala Sweden}

\section{References}

1 Amos AF, McCarty DJ, Zimmet P. The rising global burden of diabetes and its complications: estimates and projections to the year 2010. Diabet Med 1997; 14: (Suppl. 5): S1-S85.

2 Levine F, Leibowitz G. Towards gene therapy of diabetes mellitus. Mol Med Today 1999; 5: 165-171.

3 Welsh N, Bendtzen $\mathrm{K}$, Welsh $\mathrm{M}$. Expression of an insulin/interleukin-1 receptor antagonist hybrid gene in insulinproducing cell lines (HIT-T15 and NIT-1) confers resistance against interleukin-1 induced nitric oxide production. J Clin Invest 1995; 95: 1717-1722.

4 Welsh N. Gene therapy in diabetes mellitus: promises and pitfalls. Curr Opin Mol Ther 1999; 1: 464-470.

5 Grey ST et al. A20 inhibits cytokine-induced apoptosis and nuclear factor kappaB-dependent gene activation in islets. J Exp Med 1999; 90: 1135-1146.

6 Bühler L, Friedman T, Iacomini J, Cooper DKC. Xenotransplantation, state of the art, update 1999. Front Biosci 1999; 4: 416-432.

7 Deng S, Kucher T, Chen H, Brayman K. Intra-arterial delivery of adenoviral vectors to intact pancreata achieves highly efficient gene transfer to human pancreatic islets. Diabetes 1998, 47 (Suppl. 1): A67.

8 Wiest-Ladenburger $\mathrm{U}$ et al. DNA vaccination with glutamic acid decarboxylase (GAD) generates a strong humoral immune response in $\mathrm{BALB} / \mathrm{c}, \mathrm{C} 57 \mathrm{BL} / 6$, and in diabetes-prone NOD mice. Horm Metab Res 1998; 3010: 605-609.

9 Youssef S, Wildbaum G, Karin N. Prevention of experimental autoimmune encephalomyelitis by MIP-1alpha and MCP-1 naked DNA vaccines. J Autoimmun 1999; 13: 21-29.

10 Ahlgren $\mathrm{U}$ et al. Beta-cell-specific inactivation of the mouse Ipf1/Pdx1 gene results in loss of the beta-cell phenotype and maturity onset diabetes. Genes Dev 1998; 12: 1763-1768.

11 Apelqvist A et al. Notch signalling controls pancreatic cell differentiation. Nature 1999; 26: 877-881. 